\title{
Three-valued logics for incomplete information and epistemic $\operatorname{logic}{ }^{\star}$
}

\author{
Davide Ciucci $^{\star \star 1,2}$ and Didier Dubois ${ }^{1}$ \\ 1 IRIT, Université Paul Sabatier \\ 118 route de Narbonne, 31062 Toulouse cedex 9 (France) \\ 2 DISCo - Università di Milano - Bicocca \\ Viale Sarca 336 - U14, 20126 Milano (Italia)
}

\begin{abstract}
There are several three-valued logical systems. They give the impression of a scattered landscape. The majority of the works on this subject gives the truth tables, sometimes an Hilbert style axiomatization in a basic propositional language and a completeness theorem with respect to those truth tables. We show that all the reasonable connectives in three-valued logics can be built starting from few of them. Nevertheless, the issue of the usefulness of each system in relation with the third truth value is often neglected. Here, we review the interpretations of the third truth value. Then, we focus on the unknown case, suggested by Kleene. We show that any formula in three-valued logics can be encoded as a fragment of an epistemic logic (formulae of modal depth 1 , with modalities in front of literals), preserving all tautologies and inference rules. We study in particular, the translation of Kleene, Gödel, Łukasiewicz and Nelson logics. This work enables us to lay bare the limited expressive power of three-valued logics in uncertainty management.
\end{abstract}

\section{Introduction}

Classical Boolean logic has a remarkable advantage over many others: the definition of its connectives is not questionable, even if the truth values true (1) and false (0) can be interpreted in practice in different ways. Moreover, there is complete agreement on its model-based semantics. Its formal setting seems to ideally capture the "targeted reality". The situation is quite different with many-valued logics, where we replace the two truth values by an ordered set $L$ with more than two truth values. The simplest case is three-valued logic where we add a single intermediate value denoted by $\frac{1}{2}$. Naively, we might think that three-valued logic should be as basic as Boolean logic: the set $\left\{0, \frac{1}{2}, 1\right\}$ is the most simple example of bipolar scale, isomorphic to the set of signs $\{-, 0,+\}$. However, there is quite a number of three-valued logics since the extension to three values of the Boolean connectives is not unique. Worse, there is no agreement on the interpretation of this third truth value in the literature. Several interpretations of such a third truth value have been proposed. Here is a (probably not exhaustive) list:

\footnotetext{
* Appeared in LNCS 7519/2012, 147-159, DOI: 10.1007/978-3-642-33353-8_12

** Supported by FP7-Marie Curie Action (IEF) n.276158.
} 
1. Possible: the oldest interpretation due to Lukasiewicz [4]. Unfortunately, it seems to have introduced some confusion between modalities and truth values, that is still looming in some parts of the many-valued logic literature.

2. half-true: it is the natural understanding in fuzzy logic [13]: if it is true that a man whose height is $1.80 \mathrm{~m}$. is tall and it is false that a man with height $1.60 \mathrm{~m}$. is tall, we can think that it is half-true that a man whose height is $1.70 \mathrm{~m}$. is tall. Then $\frac{1}{2}$ captures the idea of borderline.

3. Undefined: this vision is typical of the studies on recursive functions modelled by logical formulae and it can be found in Kleene works [16]. A formula is not defined if some of its arguments are out of its domain. So, in this case the third truth value has a contaminating effect.

4. Unknown: in the same book, Kleene suggests this alternative interpretation of the intermediate value. It is the most usual point of view outside the fuzzy set community. Unfortunately, it suffers from the confusion between truth value and epistemic state, which generates paradoxes $[22,7]$, just like the Łukasiewicz proposal.

5. Inconsistent: in some sense, it is the dual of "unknown". Several works try to tame the contradiction by means of a truth value (Priest, Belnap, and some paraconsistent logics for instance). This standpoint has been criticized as also generating paradoxes [10,6].

6. Irrelevant: this point of view is similar to "undefined" but with the opposite effect: abstention. If a component of a formula has $\frac{1}{2}$ as truth value, the truth value of the whole formula is determined by the remaining components.

In the present work, we are interested in the fourth interpretation unknown of $\frac{1}{2}$ (so, also in the first one). The idea that unknown can be a truth value seems to originate from a common usage in natural language, creating a confusion between true and certainly true (or yet provable), false and certainly false. In the spoken language, saying "it is true" is often short for "I know that it is true". We mix up, in this way, the idea of truth per se with the assertion of truth. The latter reveals some information possessed by the speaker (its epistemic state). The value unknown is in conflict with certainly true and certainly false. It corresponds to an epistemic state where neither the truth nor the falsity of a Boolean proposition can be asserted. The truth values true (1) and false (0) are of ontological nature (which means that they are part of the definition of what we call proposition ${ }^{3}$ ), whereas unknown as well as certainly true and certainly false have an epistemic nature: they reflect a knowledge state. Mathematically, certainly true, certainly false and unknown are subsets of truth values, that is $\{1\}$, $\{0\}$ and $\{0,1\}$. The convention used here is the following: a subset $T \subseteq L$ of truth values attached to a proposition $p$ contains the truth values that are possible for $p$ in the considered knowledge state (the values outside $T$ are impossible) ${ }^{4}$. For instance, $\{1\}$ encodes certainly true since the only possible truth value is true. Mixing up true and certainly true is the same as confusing an element with a

\footnotetext{
3 and not that they represent Platonist ideals.

${ }^{4}$ Belnap[2] follows another convention: $T$ represents a conjunction of truth values. Then, $\{0,1\}$ encodes contradiction and the empty set represents unknown.
} 
singleton. The use of qualifiers such as certainly immediately suggests the use of modal logic, just as Łukasiewicz "truth value" possible does. Clearly, unknown means that true and false are possible ${ }^{5}$. Already in 1921, Tarski had the idea of translating the modalities possible and necessary into Lukasiewicz three-valued logic. The modal Possible is defined on $\left\{0, \frac{1}{2}, 1\right\}$ as $\diamond x=\neg x \rightarrow_{L} x=\min \{2 x, 1\}$ with Łukasiewicz negation and implication. In this translation, possible thus means that the truth value is at least $\frac{1}{2}$. So the question is: which of the two is the most expressive language? modal logic or three-valued logic?

To address this question, we adopt the opposite point of view: rather than trying to translate modal logic into a three-valued one, it seems more fruitful to do the converse. According to the discussion of the epistemic nature of the interpretation of $\frac{1}{2}$ here chosen, the framework of some epistemic logic looks convenient. In particular, and we will show this in the following, it is more expressive than all the three-valued logics of unknown, the interest of which proves marginal in practice.

The paper develops as follows: we recall an elementary variant of epistemic logic, sufficient for our translation. It is a fragment of the logic $\mathrm{KD}$, where we can express only Boolean propositional formulae prefixed by a modality (nesting of modalities is not allowed). It has a simple semantics in term of subsets of interpretations. We show how it is possible to translate propositions of the form "the truth value of three-valued proposition $\phi$ is in $T \subseteq\left\{0, \frac{1}{2}, 1\right\}$ " by a Boolean modal formula. In the following section, we explain that only very few connectives are required to generate all the other connectives known in three-valued logics (essentially the minimum on $\left\{0, \frac{1}{2}, 1\right\}$ and its residuated implication, as well as an involutive negation). Some three-valued logics like Eukasiewicz's can thus express all the others. In the remaining sections, we consider several three-valued logics and translate them into MEL. We show that the tautologies of one are theorems of the other and the converse. On the contrary, the converse translation is impossible: only a fragment of MEL can be translated into a three-valued logic, the one where modalities are placed only in front of literals. In particular, Tarski's translation from $\diamond \phi$ into $\neg \phi \rightarrow_{L} \phi$ is valid only if $\phi$ is a literal.

\section{Connectives in three-valued logics}

According to the discussion in the introduction, we must not use the same symbols for Boolean truth values and the ones of the three-valued logic as long as $\frac{1}{2}$ means unknown, since the latter should be seen as subsets of the former. We will use 0 and 1 in the Boolean case and $\mathbf{3}=\left\{\mathbf{0}, \mathbf{1}, \frac{1}{2}\right\}$ in three-valued logics. Since $\frac{1}{2}$ is interpreted as unknown, $\mathbf{0}, \mathbf{1}, \frac{1}{2}$ will be considered as epistemic truth-values and 0,1 as ontic ones. Moreover, we equip $\mathbf{3}$ with a total order $\leq$ : $\mathbf{0}<\frac{1}{2}<\mathbf{1}$, often referred to as the truth ordering [2].

Conjunction, implication and negation on the set of values $\mathbf{0}, \frac{1}{2}, \mathbf{1}$ can be defined by minimal intuitive properties.

\footnotetext{
${ }^{5}$ Actually, Lukasiewicz proposed this idea for the study of contingent futures: it is possible that the battle will be won or lost.
} 
Definition 1. A conjunction on $\mathbf{3}$ is a binary mapping $*: \mathbf{3} \times \mathbf{3} \mapsto \mathbf{3}$ such that

(C1) If $x \leq y$ then $x * z \leq y * z$;

(C2) If $x \leq y$ then $z * x \leq z * y$;

(C3) $\mathbf{0} * \mathbf{0}=\mathbf{0} * \mathbf{1}=\mathbf{1} * \mathbf{0}=\mathbf{0}$ and $\mathbf{1} * \mathbf{1}=\mathbf{1}$.

We note that $(\mathrm{C} 3)$ requires that $*$ be an extension of the connective AND in Boolean logic. Then, the monotonicity properties (C1-C2) imply $\frac{1}{2} * \mathbf{0}=$ $\mathbf{0} * \frac{1}{2}=\mathbf{0}$. If we consider all the possible cases, there are 14 conjunctions satisfying definition 1. Among them, only six are commutative and only five associative. These five conjunctions are already known in literature and precisely, they have been studied in the following logics: Sette [19], Sobociński [20], Łukasiewicz [4], Kleene [16], Bochvar [3]. The idempotent and commutative Kleene conjunction and disjunction (the minimum, denoted by $\sqcap$ and the maximum denoted by $\sqcup$ ) are present in $\mathbf{3}$ due the total order assumption $(x \sqcap y=y \sqcap x=x$ if and only if $x \leq y$ if and only if $x \sqcup y=y \sqcup x=y$ ).

In the case of implication, we can give a general definition, which extends Boolean logic and supposes monotonicity (decreasing in the first argument, increasing in the second).

Definition 2. An implication on $\mathbf{3}$ is a binary mapping $\rightarrow: \mathbf{3} \times \mathbf{3} \mapsto \mathbf{3}$ such that

(I1) If $x \leq y$ then $y \rightarrow z \leq x \rightarrow z$;

(I2) If $x \leq y$ then $z \rightarrow x \leq z \rightarrow y$;

(I3) $\mathbf{0} \rightarrow \mathbf{0}=\mathbf{1} \rightarrow \mathbf{1}=\mathbf{1}$ and $\mathbf{1} \rightarrow \mathbf{0}=\mathbf{0}$.

From the above definition we derive $x \rightarrow \mathbf{1}=\mathbf{1}, \mathbf{0} \rightarrow \mathbf{1}=\mathbf{1}$ and $\frac{1}{2} \rightarrow \frac{1}{2} \geq$ $\left\{\mathbf{1} \rightarrow \frac{1}{2}, \frac{1}{2} \rightarrow \mathbf{0}\right\}$. There are 14 implications satisfying this definition. Nine of them are known and have been studied. Besides those implications named after the five logics mentioned above, there are also those named after Jaśkowski [15], Gödel [12], Nelson [17], Gaines-Rescher [11]. Gödel implication is present in 3 due to the total order and using the residuation:

$$
x \sqcap y \leq z \text { if and only if } x \leq y \rightarrow_{G} z .
$$

It is such that $y \rightarrow_{G} z=\mathbf{1}$ if $y \leq z$ and $z$ otherwise.

Finally, there are only three possible negations that extend the Boolean negation, that is if $\mathbf{0}^{\prime}=\mathbf{1}$ and $\mathbf{1}^{\prime}=\mathbf{0}$ :

1. $\sim \frac{1}{2}=\mathbf{0}$. It corresponds to an intuitionistic negation (it satisfies the law of contradiction, not the excluded middle).

2. $\neg \frac{1}{2}=\frac{1}{2}$. It is an involutive negation.

3. $-\frac{1}{2}=1$. It corresponds to a paraconsistent negation (as it satisfies the law of excluded middle, not the one of contradiction).

The intuitionistic negation is definable by Gödel implication as $\sim x=x \rightarrow_{G} 0$. Finally, despite the existence of several known systems of three-valued logics, we can consider that in the above setting, there is only one encompassing threevalued structure. That is, all the connectives satisfying the above definitions, can be obtained from a structure equipped with few primitive connectives [5]. 
Proposition 1. We denote by $\mathbf{3}$ the set of three elements without any structure and by $\overline{3}$ the same set equipped with the usual order $\mathbf{0}<\frac{1}{2}<\mathbf{1}$ or equivalently, $\overline{3}=\left(\mathbf{3}, \sqcap, \rightarrow_{G}\right)$. All 14 conjunctions and implications can be expressed in any of the following systems:

$-(\overline{3}, \neg)=\left(\mathbf{3}, \sqcap, \rightarrow_{G}, \neg\right)$;

- $\left(\overline{3}, \rightarrow_{K}\right)$ where $\rightarrow_{K}$ is Kleene implication $(\max (1-x, y))$;

- $\left(\mathbf{3}, \rightarrow_{L}, \mathbf{0}\right)$ where $\rightarrow_{L}$ is Lukasiewicz implication $(\min (1,1-x+y))$;

- $\left(\mathbf{3}, \rightarrow_{K}, \sim, \mathbf{0}\right)$ where $\rightarrow_{K}$ is Kleene implication and $\sim$ the intuitionistic negation.

So, in the first two cases, we assume a residuated chain, whereas in the other two, we can derive it from the other connectives. We remark also that the intuitionistic negation can be replaced by the paraconsistent negation in the last item. The above result differs from functional completeness, since it only deals with threevalued functions that coincide with a Boolean connective on $\{T, F\}$.

\section{A simple information logic}

Admitting that the concept of "unknown" refers to a knowledge state rather than to an ontic truth value, we may keep the logic Boolean and add to its syntax the capability of stating that we ignore the truth value ( 1 or 0$)$ of propositions. The natural framework to syntactically encode statements about knowledge states of propositional logic (PL) statements is modal logic, and in particular, the logic KD. Nevertheless, only a very limited fragment of this language is needed here: the language MEL [1].

Consider a set of propositional variables $\mathcal{V}=\{a, b, c, \ldots, p, \ldots\}$ and a standard propositional language $\mathcal{L}$ built on these symbols along with the Boolean connectives of conjunction and negation $\left(\wedge,,^{\prime}\right)$. As usual, disjunction $\alpha \vee \beta$ stands for $\left(\alpha^{\prime} \wedge \beta^{\prime}\right)^{\prime}$, implication $\alpha \rightarrow \beta$ stands for $\alpha^{\prime} \vee \beta$, and tautology $\top$ for $\alpha \vee \alpha^{\prime}$. Let us build another propositional language $\mathcal{L}_{\square}$ whose set of propositional variables is of the form $\mathcal{V}_{\square}=\{\square \alpha: \alpha \in \mathcal{L}\}$ to which the classical connectives can be applied. It is endowed with a modality operator $\square$ expressing certainty, that encapsulates formulas in $\mathcal{L}$. We denote by $\alpha, \beta, \ldots$ the propositional formulae of $\mathcal{L}$, and $\phi, \psi, \ldots$ the modal formulae of $\mathcal{L}_{\square}$. In other words

$$
\mathcal{L}_{\square}=\square \alpha: \alpha \in \mathcal{L}|\neg \phi| \phi \wedge \psi|\phi \vee \psi| \phi \rightarrow \psi .
$$

The logic MEL [1] uses the language $\mathcal{L}_{\square}$ with the following axioms:

1. $\phi \rightarrow(\psi \rightarrow \phi)$

2. $(\psi \rightarrow(\phi \rightarrow \mu)) \rightarrow((\psi \rightarrow \phi) \rightarrow(\psi \rightarrow \mu))$

3. $\left(\phi^{\prime} \rightarrow \psi^{\prime}\right) \rightarrow(\psi \rightarrow \phi)$

$(\mathrm{RM}): \square \alpha \rightarrow \square \beta$ if $\vdash \alpha \rightarrow \beta$ in PL.

$(\mathrm{M}): \square(\alpha \wedge \beta) \rightarrow(\square \alpha \wedge \square \beta)$

(C) : $(\square \alpha \wedge \square \beta) \rightarrow \square(\alpha \wedge \beta)$

$(\mathrm{N})$ 
(D) : $\square \alpha \rightarrow \diamond \alpha$

and the inference rule is modus ponens. As usual, the possible modality $\diamond$ is defined as $\diamond \alpha \equiv\left(\square \alpha^{\prime}\right)^{\prime}$. The first three axioms are those of PL and the other those of modal logic KD. (M) and (C) can be replaced by axiom (K):

$$
(K): \square(p \rightarrow q) \rightarrow(\square p \rightarrow \square q) .
$$

MEL is the subjective fragment of KD (or S5) without modality nesting.

The MEL semantics is very simple [1]. Let $\Omega$ be the set of $\mathcal{L}$ interpretations: $\{\omega: \mathcal{V} \rightarrow\{0,1\}\}$. The set of models of $\alpha$ is $[\alpha]=\{\omega: \omega \models \alpha\}$. A (meta)interpretation of $\mathcal{L}_{\square}$ is a non-empty set $E \subseteq \Omega$ of interpretations of $\mathcal{L}$ interpreted as an epistemic state. We define satisfiability as follows:

- $E=\square \alpha$ if $E \subseteq[\alpha]$ ( $\alpha$ is certainly true in the epistemic state $E$ )

- $E \models \phi \wedge \psi$ if $E \models \phi$ and $E \models \psi$;

$-E \models \phi^{\prime}$ if $E \models \phi$ is false.

MEL is sound and complete with respect to this semantics [1].

We remark that in this framework, uncertainty modeling is Boolean but possibilistic. The satisfiability $E \models \square \alpha$ can be written as $N([\alpha])=1$ in the sense of a necessity measure computed with the possibility distribution given by the characteristic function of $E$. Axioms (M) and (C) lay bare the connection with possibility theory [7], as they state the equivalence between $(\square \alpha \wedge \square \beta)$ and $\square(\alpha \wedge \beta)$. We can justify the choice of this minimal modal formalism. It is the most simple logic to reason on incomplete propositional information ${ }^{6}$. We only need to express that a proposition in PL is certainly true, certainly false or unknown as well as all the logical combinations of these assertions.

\section{The principles of the translation}

We denote by $v(a) \in \mathbf{3}$ the epistemic truth value of the variable $a \in \mathcal{L}$. The assertion $v(a) \in T \subseteq \mathbf{3}$ informs about the knowledge state of a Boolean variable which we also denote by $a$. Stricto sensu, we should not use the same notation for three-valued propositional variables and Boolean ones. However, we will do it for the sake of simplicity assuming that $a$ possesses an epistemic truth value $v(a)$ and an ontic truth-value $t(a) \in\{0,1\}$. If we interpret the three epistemic truth-values $\mathbf{0}, \mathbf{1}, \frac{1}{2}$ as certainly true, certainly false and unknown respectively, we can encode the assignment of such truth-values to a propositional variable $a$ in MEL as follows. We denote by $\mathcal{T}(v(a) \in T)$ the translation into MEL of

\footnotetext{
${ }^{6}$ We can debate whether MEL is an epistemic or a doxastic logic. This formalism does not take side, since axiom D is valid in both S5 and KD45 and the axiom T of knowledge $(\square \alpha \rightarrow \alpha)$ is not expressible in MEL, which is the subjective fragment of S5 as much as a KD45 fragment. We kept the term "epistemic" in reference to the idea of an information state, whether it is consistent with reality or not.
} 
the statement $v(a) \in T$ and define it as follows, in agreement with the intended meaning of the epistemic truth-values:

$$
\begin{array}{lll}
\mathcal{T}(v(a)=\mathbf{1})=\square a & \mathcal{T}(v(a)=\mathbf{0})=\square a^{\prime} & \mathcal{T}\left(v(a)=\frac{1}{2}\right)=\diamond a \wedge \diamond a^{\prime} \\
\mathcal{T}\left(v(a) \geq \frac{1}{2}\right)=\diamond a & \mathcal{T}\left(v(a) \leq \frac{1}{2}\right)=\diamond a^{\prime} &
\end{array}
$$

We remark that these definitions shed light on the acceptability or not of the excluded middle law and the contradiction principle in the presence of the unknown value: $a$ is always ontologically true or false, but $\square a \vee \square a^{\prime}$ is not a tautology nor $\diamond a \wedge \diamond a^{\prime}$ a contradiction in MEL.

In parallel we can express 3 -valued valuations in the form of special epistemic states that serve as interpretations of the language $\mathcal{L}_{\square}$ of MEL. Given a 3-valued valuation $v$, denote by $E_{v}$, the partial Boolean model defined by $t(a)=1$ if and only if $v(a)=\mathbf{1}$ and $t(a)=0$ if and only if $v(a)=\mathbf{0}$. Such an epistemic state $E_{v}$ has a particular form: it is the set of propositional interpretations of a noncontradictory conjunction of literals $\wedge_{v(a)=\mathbf{1}} a \wedge \wedge_{v(a)=\mathbf{0}} a^{\prime}$. Conversely, for any MEL epistemic state $E$ (a disjunction of propositional interpretations) we can assign a single 3 valued interpretation $v_{E}$ :

$$
\forall a, v_{E}(a)= \begin{cases}\mathbf{1} & E \vDash \square a \\ \mathbf{0} & E \vDash \square a^{\prime} \\ \frac{1}{2} & \text { otherwise }\end{cases}
$$

The map $E \mapsto v_{E}$ is not bijective, it defines an equivalence relation on epistemic states and $E_{v}=\cup\left\{E: v_{E}=v\right\}$ is the partial Boolean model induced by $v$. Let us now consider the fragment of MEL where we can put modalities only in front of literals, that is $\mathcal{L}_{\square}^{\ell}=\square a\left|\square a^{\prime}\right| \phi^{\prime}|\phi \wedge \psi| \phi \vee \psi$.

Proposition 2. Let $\alpha$ be a formula and $\Gamma$ a set of formulae built on the language of the fragment $\mathcal{L}_{\square}^{\ell}$. Then, $\Gamma \vdash \alpha$ if and only if $\forall v, E_{v} \models \Gamma$ implies $E_{v} \models \alpha$.

In the following, we consider four known three-valued logics and show that, insofar as the third truth-value means unknown, they can be expressed in MEL: Kleene, Gödel three-valued intuitionistic, Łukasiewicz and Nelson logics. The two first ones can be viewed as fragments of the latter. Especially, we show that $\mathcal{L}_{\square}^{\ell}$ exactly characterizes any of Łukasiewicz and Nelson logics as we will see in the next sections. We start with the simplest logic.

\section{The Kleene Fragment of MEL}

The best known and often used logic to represent uncertainty due to incomplete information is Kleene logic. The connectives are simply the min $\sqcap$, the max $\sqcup$, the involutive negation $\neg$. A material implication $a \rightarrow_{K} b:=\neg a \sqcup b$ is then derived. The involutive negation preserves the De Morgan laws between $\sqcap$ and $\sqcup$. The syntax of Kleene logic is the same as the one of propositional logic (replacing $\wedge, \vee,^{\prime}$ by $\sqcap, \sqcup, \neg$ ). Besides, it is known that Kleene logic does not have any tautology (there is no formula $\alpha$ such that $\forall v, v(\alpha)=\mathbf{1}$ ). 
We first show that all the assignments of epistemic truth values to Kleene formulae, in the form $v(\alpha) \in T \subseteq \mathbf{3}$ can be translated into MEL. Using the translation of atoms in Section 4, the translation of other formulae is

$$
\begin{aligned}
\mathcal{T}(v(\alpha \sqcap \beta) \geq i) & =\mathcal{T}(v(\alpha) \geq i) \wedge \mathcal{T}(v(\beta) \geq i), i \geq \frac{1}{2} \\
\mathcal{T}(v(\alpha \sqcup \beta) \geq i) & =\mathcal{T}(v(\alpha) \geq i) \vee \mathcal{T}(v(\beta) \geq i), i \geq \frac{1}{2} \\
\mathcal{T}(v(\alpha \sqcap \beta) \leq i) & =\mathcal{T}(v(\alpha) \leq i) \vee \mathcal{T}(v(\beta) \leq i), i \leq \frac{1}{2} \\
\mathcal{T}(v(\alpha \sqcup \beta) \leq i) & =\mathcal{T}(v(\alpha) \leq i) \wedge \mathcal{T}(v(\beta) \leq i), i \leq \frac{1}{2} \\
\mathcal{T}(v(\neg \alpha)=\mathbf{1}) & =\mathcal{T}(v(\alpha)=\mathbf{0})=\left(\mathcal{T}\left(v(\alpha) \geq \frac{1}{2}\right)\right)^{\prime} \\
\mathcal{T}\left(v(\neg \alpha) \geq \frac{1}{2}\right) & =\mathcal{T}\left(v(\alpha) \leq \frac{1}{2}\right)=(\mathcal{T}(v(\alpha)=\mathbf{1}))^{\prime}
\end{aligned}
$$

The translation of Kleene implication can be obtained in this way; we can define it directly as follows using standard material implication $\rightarrow$.

$$
\begin{aligned}
& \mathcal{T}\left(v\left(\alpha \rightarrow_{K} \beta\right)=\mathbf{1}\right)=\mathcal{T}\left(v(\alpha) \geq \frac{1}{2}\right) \rightarrow \mathcal{T}(v(\beta)=\mathbf{1}) \\
& \mathcal{T}\left(v\left(\alpha \rightarrow_{K} \beta\right) \geq \frac{1}{2}\right)=\mathcal{T}(v(\alpha)=\mathbf{1}) \rightarrow \mathcal{T}\left(v(\beta) \geq \frac{1}{2}\right)
\end{aligned}
$$

If $\alpha=a, \beta=b$ are atoms, we obtain $\square \neg a \vee \square b$ and $\diamond \neg a \vee \diamond b$ respectively. The translation into MEL lays bare the meaning of Kleene implication: $a \rightarrow_{K} b$ is "true" means that $b$ is certain if $a$ is possible.

Example 1. Consider the formula $\alpha=\neg(a \sqcap(\neg(b \sqcup \neg c)))$. Then, $\mathcal{T}(v(\alpha)=\mathbf{1})=$ $\mathcal{T}(v(a \sqcap(\neg(b \sqcup \neg c)))=\mathbf{0})$. So, we get $\mathcal{T}(v(a)=\mathbf{0}) \vee \mathcal{T}(v(\neg(b \sqcup \neg c))=\mathbf{0})=\square a^{\prime} \vee$ $\mathcal{T}(v(b \sqcup \neg c)=\mathbf{1})$ and finally, $\square a^{\prime} \vee \mathcal{T}(v(b)=1) \vee \mathcal{T}(v(\neg c)=\mathbf{1})=\square a^{\prime} \vee \square b \vee \square c^{\prime}$. Note that we could more simply put $\alpha$ in conjunctive normal form as $\neg a \vee b \vee \neg c$ then put $\square$ in front of each literal.

A knowledge base $B$ in Kleene logic is a conjunction of formulae supposed to have designated truth value $\mathbf{1}$. We can always transform this base in conjunctive normal form $(\mathrm{CNF})$, that is, a conjunction of disjunction of literals (without simplifying terms of the form $a \sqcup \neg a$ ). Its translation into MEL consists of the same set of clauses, where we put the modality $\square$ in front of each literal. It is easy to see that the translation of any propositional tautology (if we replace each literal $l$ by $\square l$ in its CNF) will no longer be a tautology in MEL.

Finally we see that the fragment of MEL that exactly captures the language of Kleene logic contains only the set (conjunctions) of disjunctions of the elementary formulae of the form $\square a$ or $\square a^{\prime}: \mathcal{L}_{\square}^{K}=\square a\left|\square a^{\prime}\right| \phi \vee \psi \mid \phi \wedge \psi \subset \mathcal{L}_{\square}^{\ell}$. We remark that the modal axioms of MEL cannot be expressed in this fragment.

Nevertheless, we can use MEL to reason in Kleene logic. We note that the modus ponens applies to literals (since from $\square a$ and $\square a^{\prime} \vee \square b$, we can derive $\square b$ in MEL). The same counterpart of the resolution principle is also valid. It is like a propositional logic without tautologies but with such standard rules of inference. At the semantic level we can prove the following result.

Proposition 3. Let $\alpha$ be a formula in Kleene logic. For each model $v$ of $\alpha$, the epistemic state $E_{v}$ is a model (in the sense of $M E L$ ) of $\mathcal{T}(v(\alpha)=\mathbf{1}$ ). Conversely, 
for each model in the sense of $M E L$ (epistemic state) $E$ of $\mathcal{T}(v(\alpha)=\mathbf{1})$ the 3valued interpretation $v_{E}$ is a model of $\alpha$ in the sense that $v_{E}(\alpha)=1$.

We can easily verify the completeness of the Kleene fragment in MEL with respect to the models of the form $E_{v}$ in the sense that $\mathcal{T}(B) \vdash \mathcal{T}(v(\alpha)=\mathbf{1})$ in MEL if and only if $\forall v, E_{v} \vDash \mathcal{T}(B)$ implies $E_{v} \vDash \mathcal{T}(v(\alpha)=\mathbf{1})$.

\section{From Łukasiewicz three-valued logic to MEL and back}

Lukasiewicz three-valued logic $\mathrm{E}_{3}$ is a language powerful enough to express all connectives laid bare in section 2. It has been axiomatized by M. Wajsberg [24], using a language based on $\left(\mathcal{V}, \rightarrow_{L}, \neg\right)$, the modus ponens rule and the following axioms:

(W1) $\left(\alpha \rightarrow_{L} \beta\right) \rightarrow_{L}\left(\left(\beta \rightarrow_{L} \gamma\right) \rightarrow_{L}\left(\alpha \rightarrow_{L} \gamma\right)\right)$

(W2) $\alpha \rightarrow_{L}\left(\beta \rightarrow_{L} \alpha\right)$

(W3) $\left.\left(\neg \beta \rightarrow_{L} \neg \alpha\right) \rightarrow_{L}\left(\alpha \rightarrow_{L} \beta\right)\right)$

(W4) $\left(\left(\left(\alpha \rightarrow_{L} \neg \alpha\right) \rightarrow_{L} \alpha\right) \rightarrow_{L} \alpha\right)$

The truth-table of the implication $\rightarrow_{L}$ is given by table 1 and the involutive negation of Kleene logic is recovered as $\neg a:=a \rightarrow_{L} 0$. We can also define two

$$
\begin{array}{c|c|c|c|c|c|c|c|c}
\rightarrow_{L} & 0 & \frac{1}{2} & 1 \\
\hline 0 & 1 & 1 & 1 \\
\hline \frac{1}{2} & \frac{1}{2} & 1 & 1 \\
\hline 1 & 0 & \frac{1}{2} & 1
\end{array} \quad \begin{array}{llllll}
\odot & 0 & \frac{1}{2} & 1 \\
\hline 0 & 0 & 0 & 0 \\
\hline \frac{1}{2} & 0 & 0 & \frac{1}{2} \\
\hline 1 & 0 & \frac{1}{2} & 1
\end{array} \quad \begin{array}{llll}
\oplus & 0 & \frac{1}{2} & 1 \\
\hline 0 & 0 & \frac{1}{2} & 1 \\
\hline \frac{1}{2} & \frac{1}{2} & 1 & 1 \\
\hline 1 & 1 & 1 & 1
\end{array}
$$

Table 1. Lukasiewicz implication, conjunction and disjunction truth tables.

pairs of conjunction and disjunction connectives: $(\sqcap, \sqcup)$ and $(\odot, \oplus)$. The former pair is Kleene's, recovered as $a \sqcup b=\left(a \rightarrow_{L} b\right) \rightarrow_{L} b$ and $a \sqcap b=\neg(\neg a \sqcup \neg b)$. The other pair is $a \oplus b:=\neg a \rightarrow_{L} b$ and $a \odot b:=\neg(\neg a \oplus \neg b)$ explicitly described in Table 1. Łukasiewicz implication is translated into MEL as:

$$
\begin{aligned}
\mathcal{T}\left(v\left(\alpha \rightarrow_{L} \beta\right)=\mathbf{1}\right)= & {[\mathcal{T}(v(\alpha)=\mathbf{1}) \rightarrow \mathcal{T}(v(\beta)=\mathbf{1})] } \\
& \wedge\left[\mathcal{T}\left(v(\alpha) \geq \frac{1}{2}\right) \rightarrow \mathcal{T}\left(v(\beta) \geq \frac{1}{2}\right)\right] \\
\mathcal{T}\left(v\left(\alpha \rightarrow_{L} \beta\right) \geq \frac{1}{2}\right)= & \mathcal{T}(v(\alpha)=\mathbf{1}) \rightarrow \mathcal{T}\left(v(\beta) \geq \frac{1}{2}\right)
\end{aligned}
$$

The translation of $\mathcal{T}\left(v\left(\alpha \rightarrow_{L} \beta\right)=\mathbf{1}\right)$ is the same for all the 3 -valued residuated implications. In the case of atoms, we have $\mathcal{T}\left(v\left(a \rightarrow_{L} b\right) \geq \frac{1}{2}\right)=(\square a)^{\prime} \vee \diamond b$ and $\left.\mathcal{T}\left(v\left(a \rightarrow_{L} b\right)=\mathbf{1}\right)=\left((\square a)^{\prime} \vee \square b\right) \wedge\left((\diamond a)^{\prime} \vee \diamond b\right)=\square a^{\prime} \vee \square b \vee\left((\square a)^{\prime} \wedge \diamond b\right)\right)$ $\mathcal{T}\left(v\left(a \rightarrow_{L} b\right)=\mathbf{1}\right)$ thus means : if $a$ is certain then so is $b$ and if $a$ is possible then so is $b$. The translation of the connectives $\odot$ and $\oplus$ is:

$$
\begin{aligned}
& \mathcal{T}(v(\alpha \oplus \beta)=\mathbf{1})=\mathcal{T}(v(\alpha)=\mathbf{1}) \vee \mathcal{T}(v(\beta)=\mathbf{1}) \vee\left(\mathcal{T}\left(v(\alpha) \geq \frac{1}{2}\right) \wedge \mathcal{T}\left(v(\beta) \geq \frac{1}{2}\right)\right) \\
& \mathcal{T}\left(v(\alpha \oplus \beta) \geq \frac{1}{2}\right)=\mathcal{T}\left(v(\alpha) \geq \frac{1}{2}\right) \vee \mathcal{T}\left(v(\beta) \geq \frac{1}{2}\right)
\end{aligned}
$$


$\mathcal{T}(v(\alpha \odot \beta)=\mathbf{1})=\mathcal{T}(v(\alpha)=\mathbf{1}) \wedge \mathcal{T}(v(\beta)=\mathbf{1})$

$\mathcal{T}\left(v(\alpha \odot \beta) \geq \frac{1}{2}\right)=\left[\mathcal{T}\left(v(\alpha) \geq \frac{1}{2}\right) \wedge \mathcal{T}(v(\beta)=\mathbf{1})\right] \vee\left[\mathcal{T}(v(\alpha)=\mathbf{1}) \wedge \mathcal{T}\left(v(\beta) \geq \frac{1}{2}\right)\right]$

For the atoms, we see that $\mathcal{T}(v(a \oplus b)=\mathbf{1})=\square a \vee \square b \vee(\diamond a \wedge \diamond b)$ and $\mathcal{T}(v(\alpha \odot \beta)=$ $\mathbf{1})=\square a \wedge \square b$. Note that while the truth of Kleene disjunction $a \sqcup b$ corresponds to the requirement that one of $a$ and $b$ be certain, $a \oplus b$ is closer to the usual meaning of the disjunction, whereby $\mathcal{T}(v(a \oplus b)=\mathbf{1})$ can be true with none of $a$ or $b$ being certain. Besides, asserting the truth of a conjunction in $\mathrm{E}_{3}$ leads to the same translation for the two conjunctions. Note that in $\mathrm{E}_{3}$ the top and bottom element are translated respectively into, $\left((\square a)^{\prime} \vee \square a\right) \wedge\left((\diamond a)^{\prime} \vee \diamond a\right)$ and $\square a \wedge(\diamond a)^{\prime}$ which are indeed tautologies and contradictions in MEL, respectively. More generally:

Proposition 4. If $\alpha$ is an axiom in $\mathrm{E}_{3}$, then $\mathcal{T}(v(\alpha)=1)$ is a tautology in $M E L$.

The syntactic fragment of MEL capable of expressing $\mathrm{E}_{3}$ is: $\square a\left|\square a^{\prime}\right| \phi^{\prime} \mid \phi \wedge$ $\psi \mid \phi \vee \psi$, that is the MEL fragment $\mathcal{L}_{\square}^{\ell}$ where modalities are just in front of literals. It is clear that $\mathcal{L}_{\square}^{K}$ is a fragment of $\mathcal{L}_{\square}^{\ell}$. From $\mathcal{L}_{\square}^{\ell}$ to $\mathrm{Ł}_{3}$, we can also prove:

Proposition 5. For any formula in $\phi \in \mathcal{L}_{\square}^{\ell}$, there exists a formula $\alpha$ in $E_{3}$ such that $\phi$ is logically equivalent to $\mathcal{T}(v(\alpha)=\mathbf{1})$ in $M E L$. In particular, if $\phi$ is a MEL axiom in $\mathcal{L}_{\square}^{\ell}$, then the corresponding formula $\alpha$ is a tautology in $E_{3}$.

Proof. Sketch. We just show that there exists a translation $\theta$ from $\mathcal{L}_{\square}^{\ell}$ to Lukasiewicz logic, recursively defined as: $\theta(\square a)=a, \theta\left(\square a^{\prime}\right)=\neg a, \theta\left(\diamond a^{\prime}\right)=$ $a \rightarrow_{L} \neg a, \theta(\diamond a)=\neg a \rightarrow_{L} a, \theta(\alpha \wedge \beta)=\theta(\alpha) \sqcap \theta(\beta), \theta(\alpha \vee \beta)=\theta(\alpha) \sqcup \theta(\beta)$.

At the semantic level, Proposition 3 extends to $\mathrm{E}_{3}$. Moreover, since the sublanguage $\mathcal{L}_{\square}^{\ell}$ is exactly the Lukasiewicz fragment of MEL, we can apply Proposition 2 and obtain the completeness of this fragment of MEL with respect to the models of the form $E_{v}$ in the sense that, given a knowledge base $B_{L}$ in $\mathrm{E}_{3}$ (a conjunction of $\mathrm{E}_{3}$ formulas) $\mathcal{T}\left(B_{L}\right) \vdash \mathcal{T}(v(\alpha)=\mathbf{1})$ in MEL if and only if $\forall v, E_{v} \vDash \mathcal{T}\left(B_{L}\right)$ implies $E_{v} \vDash \mathcal{T}(v(\alpha)=\mathbf{1})$. Finally, we can prove that MEL restricted to $\mathcal{L}_{\square}^{\ell}$ is the proper target language for reasoning in $\mathrm{E}_{3}$, adopting an epistemic stance for truth-values. Indeed, from the above results we get the following.

Proposition 6. Let $\alpha$ be a formula in Lukasiewicz logic $E_{3}$ and $B_{L}$ a knowledge base in this logic. Then, $B_{L} \vdash \alpha$ in $E_{3}$ iff $\mathcal{T}\left(B_{L}\right) \vdash \mathcal{T}(v(\alpha)=\mathbf{1})$ in $M E L$.

Proof. Sketch: both MEL and Lukasiewicz logic are sound and complete. So, it is enough to show that $B_{L} \vDash_{\mathrm{E}} \alpha$ iff $\mathcal{T}\left(B_{L}\right) \vDash_{M E L} \mathcal{T}(v(\alpha)=\mathbf{1})$. One direction is the extension of Proposition 3 to the present case and the other follows by induction.

We note that all the results about Eukasiewicz logic also apply to the threevalued Nelson logic [23] $N_{3}=\left(\mathcal{V}, \sqcap, \sqcup, \rightarrow_{N}, \neg,-\right)$ due to the equivalence of the two logics. Indeed, Nelson implication is defined by Łukasiewicz implication as 
$a \rightarrow_{N} b:=a \rightarrow_{L}\left(a \rightarrow_{L} b\right)$ and Eukasiewicz implication can be defined as $a \rightarrow_{L} b:=\left(a \rightarrow_{N} b\right) \sqcap\left(\neg b \rightarrow_{N} \neg a\right)$. So, Nelson implication, once translated in the fragment $\mathcal{L}_{\square}^{\ell}$ of MEL is defined (on the atoms) as $\mathcal{T}\left(v\left(a \rightarrow_{N} b\right)=\mathbf{1}\right)=$ $(\square a)^{\prime} \vee \square b$, which says that if $\alpha$ is certain then $\beta$ is certain. This implication may look more natural in MEL than residuated ones or Kleene's.

\section{Encoding three-valued intuitionistic logic into MEL}

The three-valued Gödel logic [12] is based on the language built from the 4-tuple $\left(\mathcal{V}, \rightarrow_{G}, \sqcap, \sim\right)$, and the axioms are

(I1) $\alpha \rightarrow_{G}\left(\beta \rightarrow_{G} \alpha\right)$

(I2) $\left(\alpha \rightarrow_{G}\left(\beta \rightarrow_{G} \gamma\right)\right) \rightarrow_{G}\left(\left(\alpha \rightarrow_{G} \beta\right) \rightarrow_{G}\left(\alpha \rightarrow_{G} \gamma\right)\right.$

(I3) $(\alpha \sqcap \beta) \rightarrow_{G} \alpha$

(I4) $(\alpha \sqcap \beta) \rightarrow_{G} \beta$

(I5) $\alpha \rightarrow_{G}\left(\beta \rightarrow_{G}(\alpha \sqcap \beta)\right)$

(I6) $\alpha \rightarrow_{G}(\alpha \sqcup \beta)$

(I7) $\beta \rightarrow_{G}(\alpha \sqcup \beta)$

(I8) $\left(\alpha \rightarrow_{G} \beta\right) \rightarrow_{G}\left(\left(\gamma \rightarrow_{G} \beta\right) \rightarrow_{G}\left(\alpha \sqcup \gamma \rightarrow_{G} \beta\right)\right)$

(I9) $(\alpha \rightarrow \beta) \rightarrow((\alpha \rightarrow \sim \beta) \rightarrow(\sim \alpha))$

(I10) $\sim \alpha \rightarrow(\alpha \rightarrow \beta)$

(I11) $\alpha \sqcup\left(\sim \beta \sqcup\left(\alpha \rightarrow_{G} \beta\right)\right)$

where $\rightarrow_{G}$ is the residuum of Kleene conjunction $\sqcap, \sim$ is the intuitionistic negation, and the Kleene disjunction $\sqcup$ is retrieved as $\alpha \sqcup \beta:=\left[\left(\alpha \rightarrow_{G} \beta\right) \rightarrow_{G}\right.$ $\beta] \sqcap\left[\left(\beta \rightarrow_{G} \alpha\right) \rightarrow_{G} \alpha\right]$. The truth tables of the implication and negation are given by Table 2. Axiom (I10), due to Hosoi [14], ensures three-valuedness. The

$$
\begin{array}{c|c|c|c}
\rightarrow_{G} & 0 & \frac{1}{2} & 1 \\
\hline 0 & 1 & 1 & 1 \\
\hline \frac{1}{2} & 0 & 1 & 1 \\
\hline 1 & 0 & \frac{1}{2} & 1
\end{array} \quad \begin{array}{ll|l}
\sim & \sim & 0 \\
\hline \frac{1}{2} & 0 \\
\hline 1 & 0
\end{array}
$$

Table 2. Truth table of Gödel implication and negation.

translation $\mathcal{T}(v(\sim \alpha)=\mathbf{1})$ in MEL of Gödel negation is the same as the translation of Kleene negation. The translation $\mathcal{T}\left(v\left(\alpha \rightarrow_{G} \beta\right)=\mathbf{1}\right)$ is the same as for Łukasiewicz implication. However,

$$
\begin{aligned}
\mathcal{T}(v(\sim \alpha) & =\mathbf{0})=\mathcal{T}\left(v(\alpha) \geq \frac{1}{2}\right) \\
\mathcal{T}\left(v\left(\alpha \rightarrow_{G} \beta\right) \geq \frac{1}{2}\right) & =\mathcal{T}\left(v(\alpha) \geq \frac{1}{2}\right) \rightarrow \mathcal{T}\left(v(\beta) \geq \frac{1}{2}\right)
\end{aligned}
$$

In the case of atoms, $\mathcal{T}\left(v\left(a \rightarrow_{G} b\right) \geq \frac{1}{2}\right)=(\diamond a)^{\prime} \vee \diamond b=\square a^{\prime} \vee \diamond b$. 
We note that the top element $\top=\alpha \rightarrow_{G} \alpha$ and the bottom element $\perp=$ $\neg\left(\alpha \rightarrow_{G} \alpha\right)$ in Gödel logic translate into a tautology and to a contradiction in MEL. Their translation is the same as for Lukasiewicz logic $\mathrm{E}_{3}$. More generally, we can justify the axioms of intuitionistic logic in MEL.

Proposition 7. If $\alpha$ is an axiom of the three-valued Gödel logic, then $\mathcal{T}(v(\alpha)=$ 1) is a tautology in MEL.

Finding the syntactic fragment of MEL (or of KD) that exactly captures this three-valued logic is an open problem. It is contained in $\mathcal{L}_{\square}^{\ell}$ and includes the formulas $\left\{\square a, \square a^{\prime}, \diamond a, a \in \mathcal{V}\right\}$. However, Proposition 3 is still valid.

\section{Conclusion}

This work suggests that the multiplicity of three-valued logics is only apparent. If the third value means unknown, the elementary modal logic MEL, and more specifically its fragment where modalities appear only in front of literals, is a natural choice to encode all of these three-valued logics. In the framework of a given application, some connectives make sense, others do not and we can choose the proper fragment. The interest in our translation, which is both modular and faithful, is double:

1. Once translated into modal logic, the meaning of a formula becomes clear since its epistemic dimension is encoded in the syntax, even if in the worst case, the size of a translated formula may grow exponentially in the number of occurrences of the input variables.

2. We can better measure the expressive power of each three-valued system. In particular it shows that the truth-functionality of three-valued logic is paid by a severe restriction to expressing knowledge about literals only, and a very restrictive view of disjunction.

This work can be extended to more than 3 "epistemic" truth values. However, the target language is then a more expressive modal logic with more or less strong modalities, such as generalized possibilistic logic [9] (where the epistemic states are possibility distributions). For instance, the 5-valued so-called equilibrium logic [18] (which can encode "answer-set" programming) has been translated into generalized possibilistic logic with weak and strong necessity operators, the epistemic states being pairs of sets of nested models [8].

The idea of expressing a many-valued logic in a two-level Boolean language (one encapsulating the other) put here at work can be adapted to other interpretations of the third truth value (such as contradictory, irrelevant, etc.) by changing the target language. We can conjecture that only the case where this truth value has an ontic nature (that is half-true, admitting that truth is a matter of degree) enables to give a clear meaning to propositional languages using the syntax of Gödel, Lukasiewicz, etc. logics and to explain their violation of the Boolean axioms, as in the case of fuzzy logics. 


\section{References}

1. Banerjee, M., Dubois, D.: A simple modal logic for reasoning about revealed beliefs. In: Sossai, C., Chemello, G. (eds.) Proc. ECSQARU 2009, Verona, Italy, LNAI 5590. pp. 805-816. Springer-Verlag (2009)

2. Belnap, N.D.: A useful four-valued logic. In: Dunn, J.M., Epstein, G. (eds.) Modern Uses of Multiple-Valued Logic, pp. 8-37. D. Reidel (1977)

3. Bochvar, D.: On a three-valued logical calculus and its application to the analysis of the paradoxes of the classical extended functional calculus. History and Philosophy of Logic 2, 87-112 (1981)

4. Borowski, L. (ed.): Selected works of J. Łukasiewicz. North-Holland, Amsterdam (1970)

5. Ciucci, D., Dubois, D.: Relationships between connectives in three-valued logics. In: Proc. IPMU, Catania. CCIS, vol. 297, pp. 633-642 (2012)

6. Dubois, D.: On ignorance and contradiction considered as truth-values. Logic Journal of the IGPL 16, 195-216 (2008)

7. Dubois, D., Prade, H.: Possibility theory, probability theory and multiple-valued logics: A clarification. Ann. Math. and AI 32, 35-66 (2001)

8. Dubois, D., Prade, H., Schockaert, S.: Stable models in generalized possibilistic logic. In: Proceedings KR 2012, Roma. pp. 519-529 (2012)

9. Dubois, D., Prade, H.: Generalized possibilistic logic. In: Scalable Uncertainty Management. pp. 428-432. LNCS 6929, Springer (2011)

10. Fox, J.: Motivation and demotivation of a four-valued logic. Notre Dame Journal of Formal Logic 31(1), 76-80 (1990)

11. Gaines, B.: Foundations of fuzzy reasoning. Int. J. of Man-Machine Studies 6, 623-668 (1976)

12. Gödel, K.: Zum intuitionistischen aussagenkalkül. Anzeiger Akademie der Wissenschaften Wien 69, 65-66 (1932)

13. Hájek, P.: Metamathematics of Fuzzy Logic. Kluwer, Dordrecht (1998)

14. Hosoi, T.: The axiomatization of the intermediate propositional systems sn of gödel. J. Coll. Sci., Imp. Univ. Tokyo 13, 183-187 (1996)

15. Jaśkowski, S.: Propositional calculus for contradictory deductive systems. Studia Logica 24, 143-160 (1969)

16. Kleene, S.C.: Introduction to metamathematics. North-Holland Pub. Co., Amsterdam (1952)

17. Nelson, D.: Constructible falsity. J. of Symbolic Logic 14, 16-26 (1949)

18. Pearce, D.: Equilibrium logic. Annals of Mathematics and Artificial Intelligence 47, 3-41 (2006)

19. Sette, A.: On propositional calculus $\mathrm{p}_{1}$. Math. Japon. 16, 173-180 (1973)

20. Sobociński, B.: Axiomatization of a partial system of three-value calculus of propositions. J. of Computing Systems 1, 23-55 (1952)

21. Surma, S.: Logical Works. Polish Academy of Sciences, Wroclaw (1977)

22. Urquhart, A.: Many-valued logic. In: Gabbay, D., Guenthner, F. (eds.) Handbook of Philosophical Logic: Volume III, Alternatives to Classical Logic. Springer (1986)

23. Vakarelov, D.: Notes on n-lattices and constructive logic with strong negation. Studia Logica 36, 109-125 (1977)

24. Wajsberg, M.: Aksjomatyzacja trówartościowego rachunkuzdań [Axiomatization of the three-valued propositional calculus]. Comptes Rendus des Séances de la Societé des Sciences et des Lettres de Varsovie 24, 126-148 (1931), english Translation in $[21]$ 